Please do not remove this page

RMIT

UNIVERSITY

\title{
Flexible metasurfaces and metamaterials: A review of materials and fabrication processes at micro- and nano-scales
}

Walia, Sumeet; Shah, Charan; Gutruf, Philipp Jonas; Nili Ahmadabadi, Hussein; Chowdhury, Dibakar; Withayachumnnankul, Withawat; Bhaskaran, Madhu

https://researchrepository.rmit.edu.au/esploro/outputs/9921862345101341/filesAndLinks?institution=61RMIT_INST\&index=null

Walia, S., Shah, C., Gutruf, P. J., Nili Ahmadabadi, H., Chowdhury, D., Withayachumnnankul, W., Bhaskaran, M., \& Sriram, S. (2015). Flexible metasurfaces and metamaterials: A review of materials and fabrication processes at micro- and nano-scales. Applied Physics Reviews, 2(1), 1-14.

https://doi.org/10.1063/1.4913751

Document Version: Accepted Manuscript

Published Version: https://doi.org/10.1063/1.4913751

Repository homepage: https://researchrepository.rmit.edu.au

(c) 2015 AIP Publishing LLC

Downloaded On 2023/04/26 21:26:52 +1000 
Thank you for downloading this document from the RMIT Research Repository.

The RMIT Research Repository is an open access database showcasing the research outputs of RMIT University researchers.

RMIT Research Repository: http://researchbank.rmit.edu.au/

\section{Citation:}

Walia, S, Shah, C, Gutruf, P, Nili Ahmadabadi, H, Chowdhury, D, Withayachumnnankul, W, Bhaskaran, M and Sriram, S 2015, 'Flexible metasurfaces and metamaterials: A review of materials and fabrication processes at micro- and nano-scales', Applied Physics Reviews, vol. 2, no. 1, 011303, pp. 1-14.

See this record in the RMIT Research Repository at:

https://researchbank.rmit.edu.au/view/rmit:30062

Version: Accepted Manuscript

Copyright Statement:

(C) 2015 AIP Publishing LLC

\section{Link to Published Version:}

http://dx.doi.org/10.1063/1.4913751 


\title{
Flexible Metasurfaces and Metamaterials: A Review of Materials and Fabrication Processes at Micro- and Nano-Scales
}

\author{
Sumeet Walia,1,2,a) Charan M. Shah,1,2,a) Philipp Gutruf,1,2 Hussein Nili,1,2 Dibakar Roy \\ Chowdhury,3 Withawat Withayachumnankul,1,4 Madhu Bhaskaran,,1,2 and Sha- \\ rath Sriram, ${ }^{1,2, b)}$
}

\begin{abstract}
${ }^{1}$ Functional Materials and Microsystems Research Group, RMIT University, Melbourne, Australia; ${ }^{2}$ MicroNano Research Facility, RMIT University, Melbourne, Australia; 3College of Engineering and Computer Science, The Australian National University, Canberra, Australia; ${ }^{4}$ School of Electrical and Electronic Engineering, The University of Adelaide, Adelaide, Australia.

a) These authors contributed equally to this work

b) Address correspondence to sharath.sriram@gmail.com
\end{abstract}

\begin{abstract}
The ability to bend, stretch, and roll metamaterial devices on flexible substrates adds a new dimension to aspects of manipulating electromagnetic waves and promises a new wave of device designs and functionalities. This work reviews terahertz and optical metamaterials realized on flexible and elastomeric substrates, along with techniques and approaches to lend tunability to the devices. Substrate electromagnetic and mechanical characteristics suitable for flexible metamaterials are summarized for readers, followed by fabrication and processing techniques, and finally novel approaches used to-date to attain tunability. Future directions and emerging areas of interests are identified with these promising to transform metamaterial design and translate metamaterials into practical devices.
\end{abstract}

\section{INTRODUCTION}

Metamaterials are electromagnetic materials with engineered sub-wavelength structures that can be designed to exhibit strong coupling with the magnetic and/or electric component of an incident electromagnetic wave. ${ }^{1-9}$ This leads to their unique properties such as anomalous reflection/refraction, perfect absorption, and sub-wavelength focusing, among others. However, lack of reliable tuning techniques continues to hamper the widespread prospective applications of these materials. Tunability can be achieved by manipulating and controlling the interaction between the metamaterials and the incident waves, to modify the transmission, reflection, and absorption of the incident waves according to the desired functionalities. Although the geometrical scalability of metamaterial designs lends them the ability to operate over several decades of frequency, many terahertz applications for cloaking, ${ }^{10-14}$ sensing, ${ }^{15-22}$ super-lensing (an optical lens with resolution below diffraction limit), ${ }^{23-34}$ on-chip photonic and optoelectronic devices, ${ }^{11}, \quad 27, \quad 28, \quad 35-40$ perfect absorbers, ${ }^{41-44}$ and energy harvesting ${ }^{45,46}$ can significantly benefit if they are mechanically flexible. Flexible devices realized especially on low surface energy polymers like polydimethylsiloxane (PDMS), adhere to all surfaces in a conformal manner which allows the incorporation of passive or functional devices onto curved surfaces, epidermis, and packaging materials unlike what can be offered by rigid platforms. Rendering flexibility to metamaterials can make it possible to wrap light-weight and transparent metamaterials around objects. As such, metamaterial functionality on conformable surfaces opens up a whole new range of applications in remote sensing, security imaging and transformable optical frequency resonant devices. Additionally, the flexibility aspect can also be exploited to achieve tunable metamaterials, which is closely linked to the properties of the substrate. Additionally, the incorporation of functional metamaterials onto conformable platforms is expected to extend terahertz metamaterials from planar two-dimensional (2D) designs to three-dimensional (3D) structures. Metamaterials designed on flexible, elastomeric substrates also enable integration with non-planar surfaces. ${ }^{47-50}$ As such, the choice of substrate, especially its dielectric properties, are a key determining factor in efficiently exploiting the advantages on offer. Concurrently, the availability of such metamaterial structures can enable tuning and enhancement of transmission/reflection responses. As such, the extension of conventional micro- and nano-fabrication techniques to demonstrate metamaterials on flexible and elastomeric substrates have opened up opportunities to tune their electromagnetic properties, ${ }^{10}, 37,40,51-54$ create sensors that can be integrated easily into the human body, ${ }^{55-57}$ cloaks that can conform to non-planar surfaces, ${ }^{47,51,52,58}$ negative index materials, ${ }^{4,30,47,52}$ biological sensing, ${ }^{22,}, 40,59$ plasmonic devices, ${ }^{17}, 35,38,51,60-62$ and absorbers. ${ }^{41,58,63-65}$

Recent reviews on the topic have outlined the significant potential of metamaterials and the development of synthesis techniques. A review article on micromachined metamaterials by Liu et al. comprehensively covers micromachined tunable metamaterials and tuning capabilities via mechanical reconfiguration of the lattice. ${ }^{66}$ Techniques for tuning of metamaterials by tailoring the nearfield interaction between the structured elements and by employing nonlinear elements have also been critically reviewed elsewhere. ${ }^{9}$ Excellent review articles covering other aspects, like design, actuators, and mechanical deformation of metamaterials and their tunability exist. ${ }^{49}, 67$ ${ }^{76}$ However, to the best of our knowledge, there is a lack of a comprehensive review on flexible metamaterials 
encompassing a comprehensive overview of the substrate properties, processing, and tuning techniques.

In this article, flexible and elastomeric substrates used to tune the resonance frequency of metamaterials will be reviewed. Electromagnetic and mechanical properties of some of the commonly used flexible substrates and polymers will be compared and critically reviewed. Recent progress in fabrication techniques for realizing flexible metamaterials, with recent 3D approaches, will be assessed. Finally, an outlook for future directions and applications based on elastic, reversible tuning of metamaterials are presented.

\section{FLEXIBLE SUBSTRATES METAMATERIALS}

FOR

Flexible substrates provide ideal platforms for exploring some of the unique characteristics that arise in metamaterials via mechanical deformation. The use of flexible substrates to demonstrate metamaterials with novel functionalities is gaining increasing attention worldwide. $^{10,18,22,30,40,47,51,52,54,65,77-85}$ These elastic substrates are of special interest owing to their natural ability to achieve large frequency tunability through mechanical deformation, ${ }^{86}$ thereby eliminating the need to integrate an external actuator or bias voltage to achieve desired tuning. ${ }^{48,60,87}$ The resonator structures fabricated on such elastic substrates exhibits high sensitivity to structural parameters, which allow small changes in dimensions to be sensed easily. Such mechanical tuning of metamaterials has been used to demonstrate wireless strain sensing, ${ }^{19,88}$ biological sensing, ${ }^{22,} 40,59$ and absorbers, ${ }^{41,58,89}$

The most commonly used flexible substrates for metamaterials are polydimethylsiloxane (PDMS) and polyimide, due to their widespread use in flexible electronics. Other flexible substrates utilized for metamaterial devices include metaflex, ${ }^{22,79}$ polyethylene naphthalene (PEN) ${ }^{20}$, 78, 80 polyethylene terephthalate (PET), ${ }^{83}, \quad 84, \quad 90$ polymethylmethacrylate (PMMA), ${ }^{90}$ and polystyrene. ${ }^{91}$

\section{Properties of Flexible Substrates for Metamaterials}

The intrinsic properties of the substrate (dielectric permittivity, loss tangent, refractive index and Young's modulus) play a critical role in the operation of metamaterials. A suitable flexible substrate can be chosen based on the application and operating frequency. This section presents a brief overview of the desired electromagnetic and mechanical properties of flexible substrates that make them suitable for the realization of metamaterials.

\section{Electromagnetic properties}

Although metamaterials inherit their electromagnetic properties from the design of sub-wavelength resonators, the constituting media influence performance. To optimize the performance, the choice of the substrate is governed by the following desired characteristics: (i) low permittivity, to minimize the capacitive effect in metamaterials and enhance the $Q$-factor and (ii) low loss (absorption coefficient) to maximize transmission through or propagation along the substrate. A low refractive index is preferable to minimize reflection losses arising from the substrate. The refractive index $(n)$ and the dielectric permittivity $(\varepsilon)$ are interrelated by the expression $n=\sqrt{ } \varepsilon$. Some of the important electrical properties of commonly used polymer substrates to realize metamaterials are summarized in Table I.

\section{Processability and mechanical properties}

Polymers offer widespread opportunities for the creation of versatile, large-area, and low-cost fabrication of flexible metamaterials. A wide variety of polymeric substrates with different mechanical and electrical properties are being investigated to design flexible metamaterial devices that are capable of operating from the microwave (a few $\mathrm{GHz}$ ) through to optical frequencies (down to visible and ultraviolet wavelengths of about $300 \mathrm{~nm}$ ). These flexible polymers can be easily processed using spincoating, thermal curing, and well-established microfabrication techniques to generate a substrate of choice for metamaterials. Micro/nano-fabrication techniques such as lithography patterning (contact, optical, soft, and shadow mask), laser writing, molding, casting, and transfer have all been demonstrated for polymer-based metamaterials, and will be discussed in detail later.

The mechanical properties of the substrates (benchmarked by their Young's modulus) are critical for determining their viability for the development of mechanically tunable metamaterials. Substrates with low Young's modulus can undergo large mechanical deformations with high level of reversibility and repeatability that can allow significant modulation of the fundamental and higher order resonance modes. However, fabrication processes impose certain limitations over the choice of flexible substrates. These include a requirement of high operating temperatures for deposition and annealing, and the presence of highly planar surfaces to undertake lithography or similar patterning techniques. Table I lists the mechanical properties of some of the popular polymer substrates for metamaterials. Based on the application and regime of operational frequency, a flexible substrate with low absorption loss $(\alpha)$ (see Table I) and the desired mechanical properties can be chosen.

\section{POLYMERS FOR METAMATERIAL FABRICATION}

While a diverse range of polymers have been utilized (see Table I), three materials are repeatedly utilized by researchers due to certain favorable characteristics. This section discusses the salient features and limitations of these materials.

\section{Polydimethylsiloxane}

Polydimethylsiloxane (PDMS) is an elastomeric polymer with unique attributes like low surface energy, biocompatibility, and excellent flexibility and elasticity. Normal operating temperatures of PDMS range from $50{ }^{\circ} \mathrm{C}$ to $200{ }^{\circ} \mathrm{C} .{ }^{92}$ Being a soft elastomeric polymer, PDMS can be easily integrated with non-planar structures with a high degree of conformability. Its compatibility with conventional and advanced micro/nano-fabrication techniques such as soft lithography and imprint lithography are added advantages. ${ }^{56,93}$ Properties such as the low Young's modulus $\left(7.5 \times 10^{-4} \mathrm{GPa}\right)^{94}$ and low absorption losses $\left(13 \mathrm{~cm}^{-1}\right.$ at $\left.1 \mathrm{THz}\right)$ (Table I) are attributes that 
make PDMS a suitable substrate for flexible, tunable metamaterials. Its highly elastic nature (up to $\sim 120 \%$ reversible stretchability), makes it a viable substrate for tuning metamaterials via mechanical deformations. Furthermore, its freestanding nature and good transparency across a broad range of the electromagnetic spectrum make it suitable for wide bandwidth metamaterial applications. The low surface energy 
TABLE I. Electromagnetic, electrical, and mechanical properties of polymers commonly used as flexible substrates.

\begin{tabular}{|c|c|c|c|c|c|c|c|c|c|c|}
\hline Material & $\begin{array}{c}\text { Dielectric } \\
\text { Permittivity } \varepsilon \\
(0.2-2.5 \text { THz) }\end{array}$ & $\begin{array}{c}\text { Loss } \\
\text { Tangent } \\
\tan \delta\end{array}$ & $\begin{array}{c}\text { Absorption } \\
\text { Coefficient } \\
\alpha\left(\mathrm{cm}^{-1}\right) \\
(\text { at } 1 \mathrm{THz})\end{array}$ & $\begin{array}{l}\text { Resistivity } \\
\rho(\Omega \mathrm{cm})\end{array}$ & $\begin{array}{l}\text { Young's } \\
\text { Modulus } \\
\text { E (GPa) }\end{array}$ & $\begin{array}{c}\text { Operating } \\
\text { Temperature } \\
\left({ }^{\circ} \mathrm{C}\right)\end{array}$ & $\begin{array}{l}\text { Curing Condi- } \\
\text { tions }\end{array}$ & $\begin{array}{l}\text { Glass Transi- } \\
\text { tion Temper- } \\
\text { ature } T_{\mathrm{g}}\left({ }^{\circ} \mathrm{C}\right)\end{array}$ & Planarization & References \\
\hline $\begin{array}{l}\text { Polydimethyl- } \\
\text { siloxane (PDMS) }\end{array}$ & 2.35 & $\begin{array}{l}0.020- \\
0.060\end{array}$ & 13 & $2.9 \times 10^{14}$ & $7.5 \times 10^{-4}$ & -45 to 200 & $\begin{array}{c}27{ }^{\circ} \mathrm{C}, 24 \mathrm{~h} \text { or } \\
70^{\circ} \mathrm{C}, 1 \mathrm{~h}\end{array}$ & -125 & Moderate & 53,94 \\
\hline Polyimide & 3.24 & 0.031 & 12 & $1.7 \times 10^{17}$ & 2.5 & -269 to 400 & $180^{\circ} \mathrm{C}, 30 \mathrm{~min}$ & 350 & Good & 95 \\
\hline $\begin{array}{l}\text { Polyethylene tereph- } \\
\text { thalate (PET) }\end{array}$ & 2.86 & $\begin{array}{c}0.053- \\
0.072\end{array}$ & 25 & & 4.0 & -80 to 180 & & 80 & Excellent & 96,97 \\
\hline $\begin{array}{l}\text { Polyethylene } \\
\text { napthalate (PEN) }\end{array}$ & 2.56 & 0.003 & 1 & & 5.2 & & & 150 & Excellent & 96 \\
\hline $\begin{array}{l}\text { Benzocyclobutene } \\
\text { (BCB) }\end{array}$ & 2.65 & $\begin{array}{l}0.001- \\
0.009\end{array}$ & 3 & & 2.9 & & $250^{\circ} \mathrm{C}, 1 \mathrm{~h}$ & $>350$ & Excellent & 98,99 \\
\hline $\begin{array}{l}\text { Polymethylmeth- } \\
\text { acrylate (PMMA) }\end{array}$ & 2.22 & $\begin{array}{c}0.042- \\
0.070\end{array}$ & 22 & $5.5 \times 10^{4}$ & 3.1 & & $180{ }^{\circ} \mathrm{C}, 2 \mathrm{~min}$ & 105 & Excellent & 99 \\
\hline Polypropylene & 2.25 & 0.008 & 2 & $1.0 \times 10^{13}$ & 2.0 & 0 to 135 & & 170 & Moderate & \\
\hline Paralyene & 3.00 & 0.120 & & $8.8 \times 10^{16}$ & & 80 & & 290 & Excellent & \\
\hline SU8 & 2.89 & 0.140 & 25 & $5.1 \times 10^{16}$ & & 200 & & 210 & Excellent & \\
\hline Polystyrene & 2.53 & & 11 & $>10^{16}$ & 3.1 & 65 & & 107 & Poor & \\
\hline
\end{tabular}




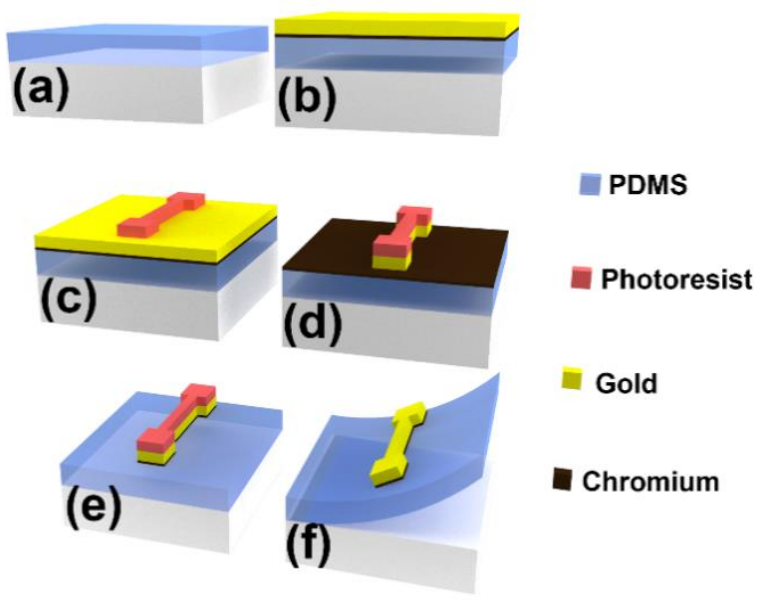

FIG. 1. Schematic representation of a fabrication sequence for flexible elastomer-based microdevices. (a) The elastomer substrate (PDMS) is defined by spin-coating onto a carrier substrates for fabrication. (b) Metal thin films are deposited, for example, gold with a chromium adhesion layer. (c-e) The metal layers are patterned by photolithography and etching to define structures such as resonators. (f) Following photoresist removal, the elastomeric substrate and the defined pattern are released (by peeling off) from the carrier substrate.

of PDMS is another feature that has been exploited to efficiently transfer transparent oxides such as indium tin oxide and zinc oxide, which demonstrate excellent stability under large strains providing potential mechanisms for oxide-based tuning of electromagnetic devices. ${ }^{100}$ The possibility of imprinting structures on to PDMS opens up tremendous opportunities for designing multilayered, 3D metamaterials ${ }^{10,12}$ which can be used to create complex resonators.

PDMS has a significantly high thermal expansion coefficient (TCE) of $3.1 \times 10^{-4} /{ }^{\circ} \mathrm{C},{ }^{101}$ which can cause deposited metal thin films to define resonators or waveguides to be buckled or have surface micro-wrinkles. This can be overcome by careful control of sample temperature during deposition or the addition of an encapsulation that equalizes stress. A recent article predicts that the selfordered patterns of buckled metal films obtained on PDMS will find applications in optical and strain analysis devices. ${ }^{102}$

Despite some of its highly desirable features, PDMS suffers from a high temperature sensitivity (due to its large TCE) that can cause changes to the geometry of metamaterials even with small variations in temperature. Additionally, the hydrophobic surface properties of PDMS causes striations when applying photoresists, requiring additional treatments (such as surface activation by plasma) to carry-out micro-fabrication processes especially those structures with multiple layers. ${ }^{103} 104,105$

\section{Polyimide}

Polyimide (often referred to as Kapton, its commercial variant) is a widely used plastic substrate for electronic devices, such as flexible solar cells, ${ }^{106}$ interconnects, ${ }^{92}$ and metamaterials. ${ }^{19,} 41,85$ They have been demonstrated for creating flexible, free-standing metamaterials operating at terahertz frequencies to demonstrate properties such as unnaturally high refractive index ${ }^{4}$ and dual-band near-perfect absorbers. ${ }^{41}$

Polyimide exhibits an intrinsic Young's modulus of 2.5 GPa (see Table I) ${ }^{95}$ and is compatible with standard microfabrication processes. Its potential for fabricating flexible metamaterials is aided by its strong adhesion to metal coatings, which provides a high degree of strain delocalization. ${ }^{107}$ Normal operating temperatures of polyimide range from $-269{ }^{\circ} \mathrm{C}$ to $400{ }^{\circ} \mathrm{C}$ with a very high glass transition temperature (see Table I), which makes it ideal for direct deposition of metals at high temperatures using physical vapor deposition techniques such as sputtering, electron beam evaporation, and pulsed-laser deposition. Furthermore, its low thermal coefficient of expansion makes it compatible to metals or oxides of any kind, even at elevated temperatures. Its adhesion to photoresist and resistance to corrosive acids used in etching thin-film metals in microfabrication processes is another characteristic feature that allows direct patterning of highresolution configurations using conventional microfabrication techniques that are required to fabricate terahertz metamaterials. ${ }^{108}$ However, its low elastic regime, (limited by $\sim 4 \%$ strain) due to its high Young's modulus, limits the magnitude of tunability of terahertz metamaterials that can be achieved through mechanical deformations. A relatively high temperature $\left(\sim 400{ }^{\circ} \mathrm{C}\right)$ required to crosslink the precursor polymer may cause complications with some materials. ${ }^{109}$

\section{Polyethylene terephthalate}

Polyethylene terephthalate (PET) is another flexible substrate that has been explored to design complex electronic systems, such as RFID stickers, LCD display covers, and capacitive touch sensor arrays. ${ }^{110-112}$

The relatively high dielectric permittivity $(2.86),{ }^{113}$ wide operating temperature range $\left(-80{ }^{\circ} \mathrm{C}\right.$ to $\left.180{ }^{\circ} \mathrm{C}\right),{ }^{97}$ relatively high glass transition temperature $\left(78^{\circ} \mathrm{C}\right),{ }^{114} \mathrm{a}$ low thermal expansion, and strong adhesion to photoresists and metals make PET a viable choice as a substrate for creating flexible metamaterials (see Table I). PET films are planar and transparent at visible wavelengths and its electromagnetic properties in the terahertz regime is similar to that of PDMS. The aforementioned characteristics render PET with a combination of attributes that are present in PDMS and polyimide, without some of their associated limitations. However, PET is relatively high cost and susceptible to shear heat.

As a result, PET is already being investigated to create metamaterials for flexible plasmonic structures operating at near-infrared frequencies, ${ }^{115}$ and their tuning via mechanical deformations. ${ }^{115}$

\section{PROCESSING AND FABRICATION}

The fabrication techniques for metamaterials have reached high levels of sophistication, which impart the capability to synthesize metamaterials across micrometer and nanometer scales even on unconventional substrates. 

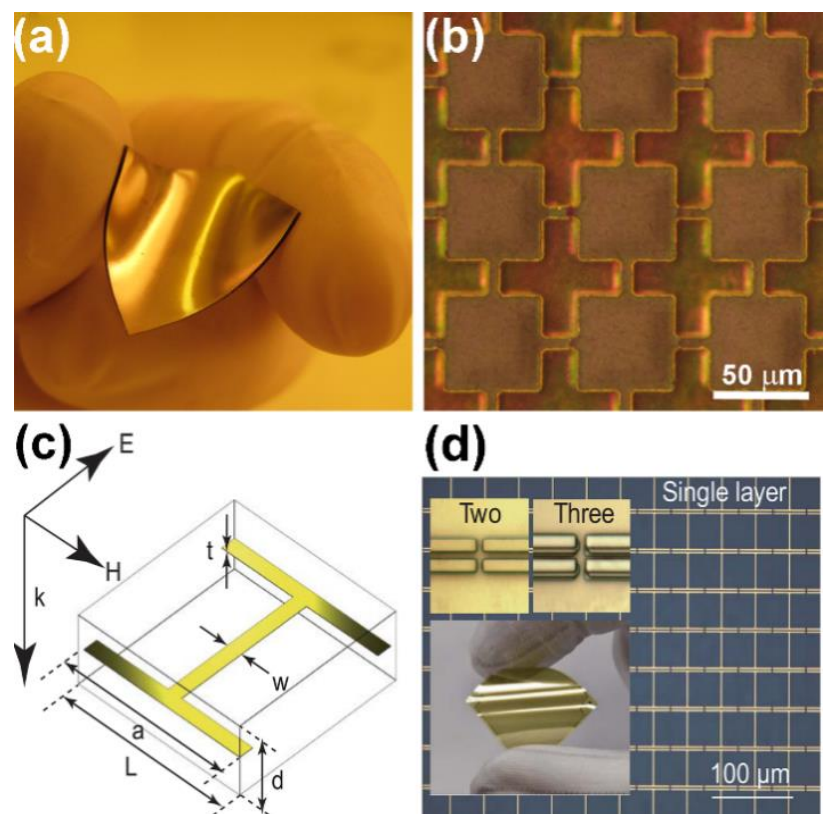

(d)

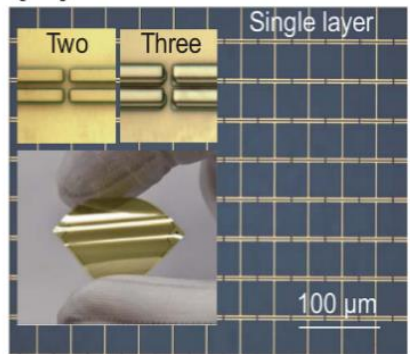

FIG. 2. Examples of microfabricated terahertz metamaterials on flexible substrates. (a,b) A multilayered, large-area fishnet structure on PDMS. Image reused with permission from Ref. 53. (c,d) 'I'-shaped resonators and dipole structures fabricated on spin-coated polyimide substrates. Image reproduced with permission from Nature 470, 369 (2011). Copyright 2011 Nature Publishing Group

The emergence of high resolution nanofabrication methods such as nanostencil lithography (NSL) has enabled nanopatterning on a wide range of flexible substrates in a single step which has contributed to the emergence of non-planar metamaterial and photonic systems. The increasing knowledge about the integration of hard materials, such as metals or dielectrics onto flexible materials coupled with the development of advanced techniques to realize nanometer feature sizes have enabled flexible and stretchable devices across multidisciplinary domains such as photonics, electronics and optoelectronics. Such developments across several disciplines has enabled rapid progress and refining of synthesis techniques which has allowed the creation of flexible metamaterials that are capable of operating across a wide and tunable frequency spectrum. ${ }^{18,22,51-53,116-119}$ These techniques have enabled the development of novel electromagnetic devices, triggering new scientific and technological developments in the field of sensing. A typical example of microfabrication process schematic to develop metamaterials operating at terahertz frequencies is shown in Figure 1.

To allow enhanced functionality and control over fabrication parameters, flexible substrates are prepared by combining the polymer with a silicon carrier substrate by spin coating, laminating, extruding, or doctor-blading. The carrier (silicon) substrate is merely used to provide mechanical strength for undertaking the microfabrication processes. During the process of combining the polymer with the carrier, it is important to obtain a smooth film that is free from trapped oxygen/air bubbles, striations, and edge beads which would otherwise interfere with the microfabrication process and/or device performance.

The transfer of resonator patterns onto flexible substrates have been reported widely in literature using several established techniques, like conventional lithogra- phy, ${ }^{53,} 88$ shadow mask lithography, ${ }^{51}$ electron beam lithography ${ }^{11}$ laser micro-lens array lithography, ${ }^{80}$ electroplating, ${ }^{120}$ and direct laser writing. ${ }^{121}$ This section reviews the prevalent processing techniques.

\section{Photolithography}

Microfabrication is a conventional technique that can be employed to synthesize metamaterials that operate at terahertz frequencies. The ability to create high-resolution 


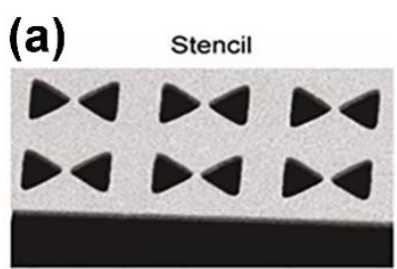

(c)

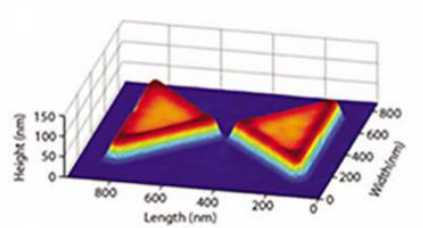

(b) Nanostencil Lithography

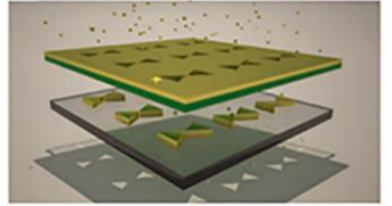

(d) Flexible

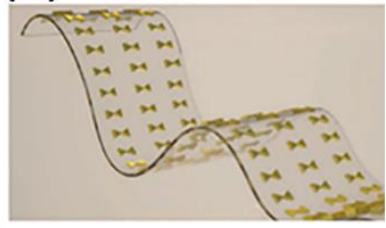

FIG. 3. Fabrication sequence in the shadow mask lithography (SML) technique. (a) A stencil defined with the pattern of interest, with bow tie antennas in this case with the side of the triangles of $500 \mathrm{~nm}$. (b) Deposition through the stencil either with a small gap or in contact. (c) Atomic force microscopy profile of a nanostructure fabricated by SML. (d) Representation of a flexible devices realized using SML. Image reproduced with permission from Adv. Mater. 23, 4422 (2011). Copyright 2011 Wiley-Blackwell

sub-wavelength structures operating at terahertz frequencies (corresponding to wavelengths of $30 \mu \mathrm{m}$ to $3 \mathrm{~mm}$ ) with minimal complications make this technique well suited for terahertz metamaterials and has been extensively used to fabricate single layer and multilayer (3D) metamaterials. ${ }^{70} 10-12,18,50,52,53,77,83,88,91,120-124$ Figure 2 shows examples of the reported metamaterials made on flexible substrates using the microfabrication technique. Figure $2(\mathrm{a}, \mathrm{b})$ shows a multilayered fishnet metamaterial developed using a microfabrication process on PDMS. The resonators were patterned into the metal $(200 \mathrm{~nm}$ gold thin films, with an adhesion layer) deposited on spincoated PDMS substrates post curing. Often, micron scale resolution patterns obtained by this technique are further encapsulated with a thin layer of PDMS to avoid delamination of metal layers.

However, surface modification of substrate from hydrophobic to hydrophilic by strong plasma treatment may be needed to make the substrate compatible with microfabrication process, ${ }^{103}$ as the hydrophobic nature of some flexible substrates make it implausible to carry out standard microfabrication processes such as uniform spin coating of photoresist films. ${ }^{103}$ However, such surface treatments are usually effective only for short durations and striations can still occur when spin coating the photoresist film. ${ }^{125}$ In addition, microfabrication techniques are suitable only for polymers that can withstand organic and corrosive solvents.

Hence, alternate micromachining methods such as soft lithography, shadow mask lithography, pattern and transfer techniques are gaining interest for obtaining metamaterial resonator patterns on flexible substrates. ${ }^{10,53,61 \text {, }}$ $91,117,126,127$ 
Stamp fabrication

(a)

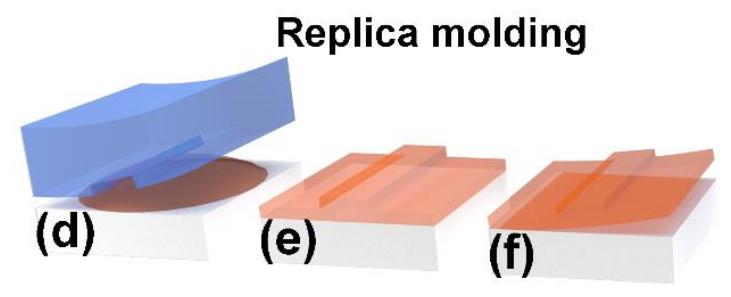

(b)

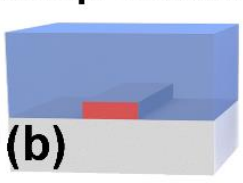

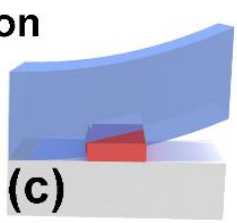

Transfer printing

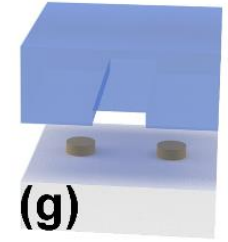

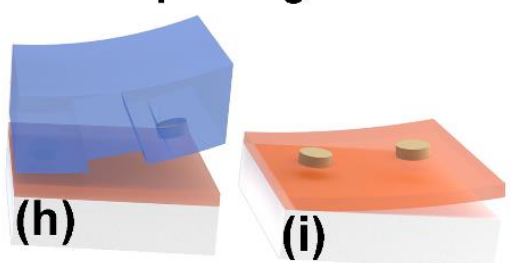

(i)

\title{
PDMS $\approx$ Photoresist $\backsim$ Substrate $\approx$ Features for transfer $\backsim$ Carrier/Host stubstrate
}

\begin{abstract}
FIG. 4. Illustrated schematics of two common soft lithography processes. (a-c) Elastomeric stamp fabrication: (a) Preparation of photographically-defined features on a silicon 'master' substrate; (b) Casting of PDMS with subsequent curing; and (c) Release of the 'stamp'. (d-f) Replica molding: (d) Preparation of the final substrate material via drop-casting, spin coating, or doctor-blading onto a carrier substrate, with subsequent imprinting using the PDMS stamp; (e) Cured inverted replica of the stamp; and (f) Release of imprinted substrate material from the carrier substrate. (g-i) Transfer printing: (g) Features defined on a host substrate are picked up using a PDMS stamp; (h) Transfer of the features onto the desired final substrate material; and (i) Release of the substrate with transferred patterns

from

carrier substrate.
\end{abstract}

\section{Shadow Mask Lithography}

Shadow mask lithography is an acid-free fabrication technique employed to create planar and multiple layer micro- and nano-scale features. ${ }^{128,129,117}$ This technique involves direct deposition on to a substrate through a stencil during the deposition of thin metal films or oxides without the need of any photolithography and etching processes. This is analogous to the screen printing approaches utilized in the manufacture of $\mathrm{T}$-shirts.

A brief description of the technique is as follows. A stencil is prepared [Fig. 3(a)], usually made by throughsubstrate etching of a silicon wafer or an aluminum foil. ${ }^{126}$ The stencil is placed either in direct contact or in close proximity to the substrate [Fig. 3(b)]. Subsequently metal or dielectric layers as required are deposited, predominantly by electron beam evaporation, to utilize its inherent line-of-sight deposition characteristics to create a 1:1 copy of the stencil on the substrate. Using the shadow mask lithography, features as small as $100 \mathrm{~nm}$ wide can be patterned on arbitrary substrates including polymers and plastics that are mechanically fragile and/or chemically sensitive [Figs. 3(c,d)]. ${ }^{51}$ This approach enables large area nanopatterning with high throughput. The stencils are reusable and demonstrate repeatable results with high-throughput replication of patterns. ${ }^{117}$ However, the resolution of the realized patterns often deteriorate over repeated cycles of deposition due to the contact and proximity placement of stencil with the substrate during the deposition.

\section{Soft Lithography}

Soft lithography is an alternative fabrication technique, which allows multi-scale patterning of micro- and nanoscale patterns on polymers. Over the years many different variants of this technique have been developed. Here we discuss some of the prevalent techniques for metamaterial fabrication $56,61,130,131$ Soft lithography is an inexpen- sive technique that can overcome some of the disadvantages encountered during photolithography. These include patterning structures below the diffraction limit and the need for high energy radiation.. The soft lithography process typically requires an elastomeric stamp which is fabricated by casting PDMS onto a 'master' structure [Figs. 4(a,b)]. The master features can be defined using the appropriate lithography technique for the pattern size. The stamp fabrication is completed by peeling off the PDMS with the embedded features of the master [Fig. 4(c)].

Replica molding allows for high resolution patterning of any polymer compatible with the PDMS stamp which even include polymers that are not photolithographically definable. ${ }^{132}$ The pre-polymer is simply applied onto a carrier [Fig. 4(d)] and the stamp pressed firmly onto the surface. Post curing and removal of the stamp, an inverted copy of the stamp is fabricated, that can be removed from the carrier to form a free standing design [Figs. 4(e,f)]

Another prevalent fabrication method is transfer printing. ${ }^{133}$ Here, features of any desired material such as semiconductors, functional oxides or metals can be prepared on a silicon substrate. This allows for the use of established patterning techniques and high process temperatures. Subsequently, the patterns are 'picked up' by the elastomeric stamp [Fig. 4(g)] and placed onto the substrate of choice [Fig. 4(h)]. The substrate can then be released from the carrier [Fig. 4(i)]. The transfer printing method is very powerful but requires precise control over the adhesion of the features to the donor substrate, the stamp as well as the target substrate. 


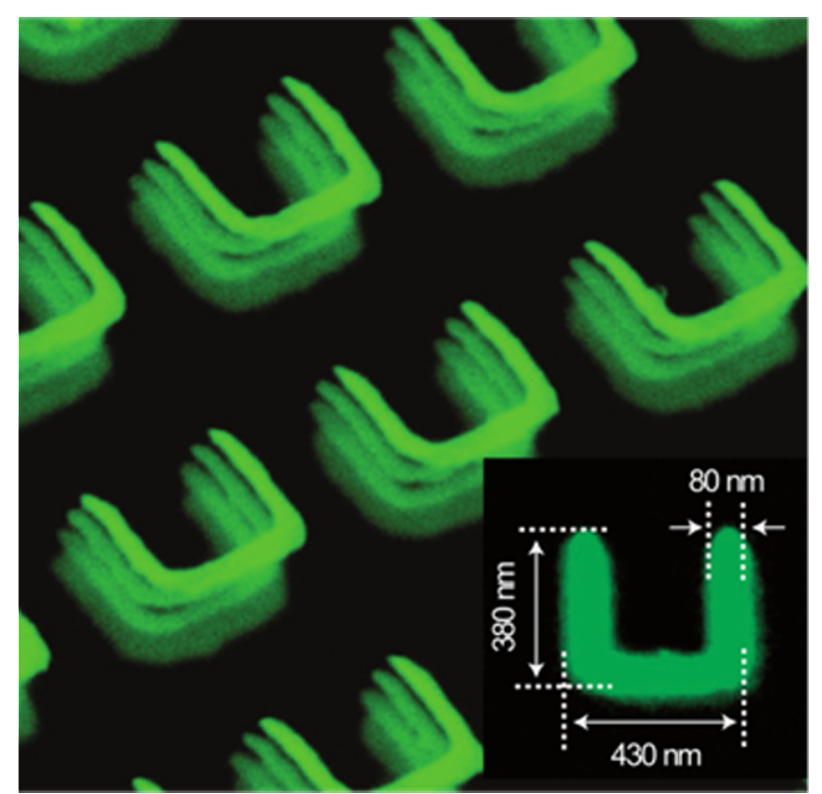

FIG. 5. A multilayered metamaterial comprising of 4 layers of gold SRRs planarized with PC403 spacer layer defined by electron beam lithography. Image reproduced with permission from Science 315, 1686 (2007). Copyright 2007 American Association for the Advancement of ScienceBy using soft lithography techniques, limitations of plastic substrates such as high temperature expansion, poor adhesion, low processing temperature and chemical instabilities can be overcome. Additionally, these techniques are suitable for a wide range of structure sizes and enable patterning on non-planar surfaces. ${ }^{52,61,134}$

\section{Electron Beam Lithography}

Electron beam lithography (EBL) uses the extremely small wavelength of a beam of electrons at high accelerating voltages to obtain nanoscale patterns. Analogous to conventional photolithography, where a photoresist layer is exposed to ultra-violet (UV) light, the EBL technique requires the exposure of an electron beam resist, polymethylmethacrylate (PMMA) or ZEP, by a highenergy electron beam. This causes a scission in the organic structure that is subsequently developed using a standard developing solution, whereby the exposed region dissolves completely in the developer. Subsequently, deposition of metal and/or dielectric layers is carried out following which the resist is dissolved to obtain the nanoscale patterns. This approach utilizes the 'lift-off' process, and so, the initial pattern defined by EBL needs to be the inverse of the desired pattern. EBL provides the highly desirable ability to create nanoscale features below the diffraction limit of the standard photolithography processes and does not require a physical mask to transfer the patterns. For metamaterials, the EBL technique is employed to fabricate sub-wavelength resolution resonators operating at optical wavelengths. ${ }^{11,48}$ Figure 5 shows a multilayered resonator structure developed on a photoconductive polymer (PC403) substrate using the EBL technique.

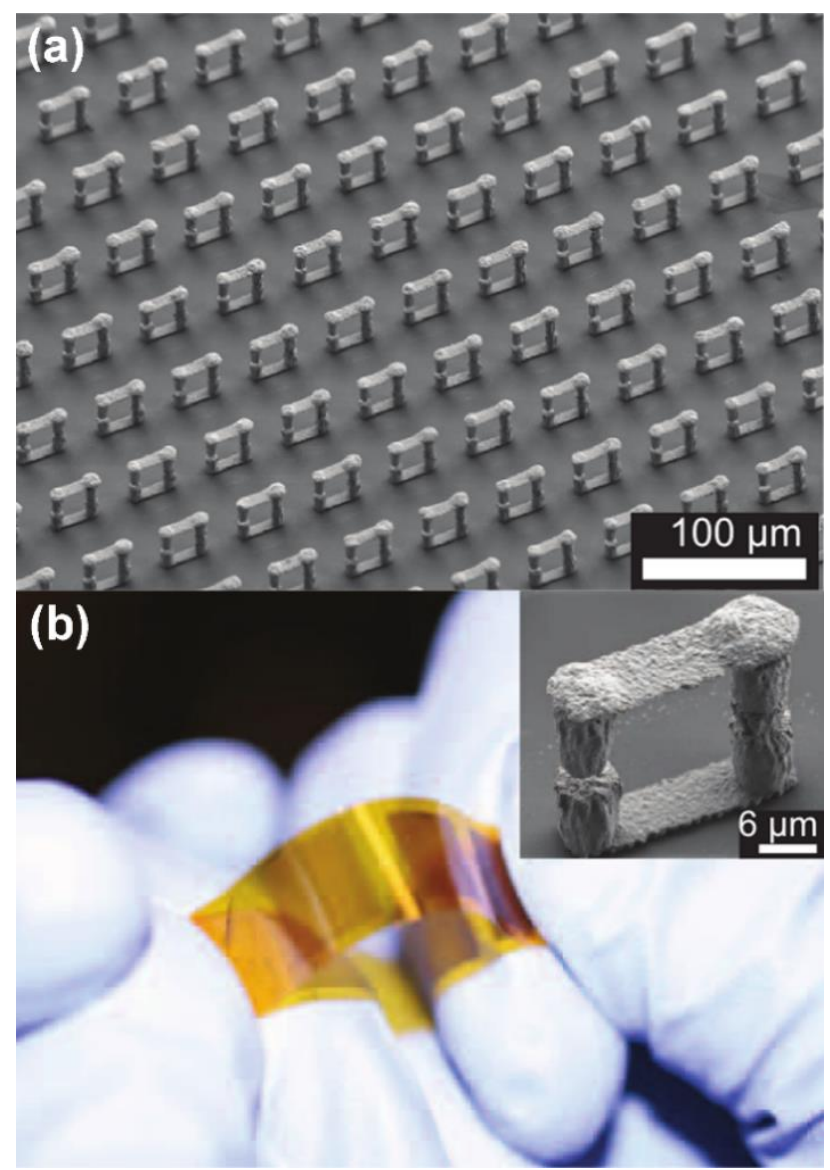

FIG. 6. (a) Scanning electron micrograph showing vertical, 3D magnetic terahertz metamaterials defined on polyimide substrates. (b) Photograph of the flexible device with an inset showing an individual 3D structure. Image reproduced with permission from Opt. Exp. 19, 12619 (2011). Copyright 2011 Optical Society of America

While EBL is a powerful technique to achieve nanometer and sub-micron features, three major limitations need to be overcome in creating large area, high performance metamaterials. These are long writing times due to the serial nature of the technique, stitching errors impacting periodicity, and the low stability and potential astigmatism of the electron beam.

Significantly longer writing time is required even for small area patterning as the process is carried out serially, one element at a time leading to reduced throughput. For example, a write area of $3 \mathrm{~mm} \times 3 \mathrm{~mm}$ would take up to $24 \mathrm{~h}$. Additionally, the serial patterning process gives rise to issues such as instability of the beam due to drifting. Large area patterning with multiple stage moves results in poor resolution and stitching defects. Secondly, stitching errors also hamper the ability to repeat the patterns over a large area without any misalignment. Finally, the stability and accuracy of the electron beam blanking as well as astigmatism are important factors that influence the effectiveness of this technique. The beam blanker is an external voltage source that is used to switch the electron beam 'ON' and 'OFF' while patterning the nanoscale features. During the 


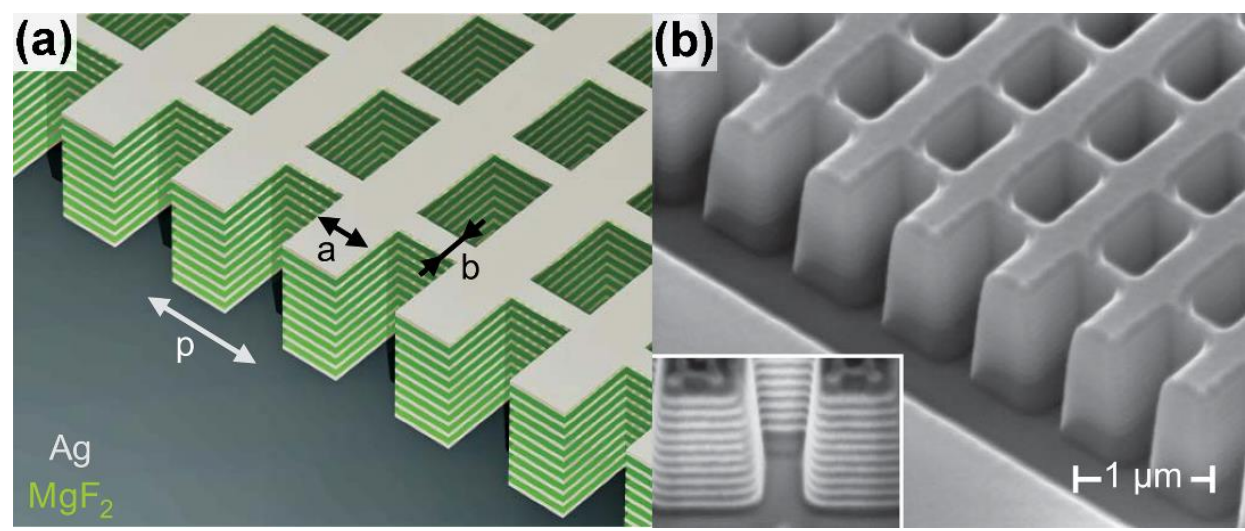

FIG. 7. Multilayered fishnet structure defined by focused ion beam milling of 21 layers -11 of silver and 10 of magnesium fluoride. The design where $p=860 \mathrm{~nm}, a=565 \mathrm{~nm}$, and $b=265 \mathrm{~nm}$ is shown in (a) with a scanning electron micrograph of the final structure shown in (b). Image reproduced with permission from Nature 455, 376 (2008). Copyright 2008 Nature Publishing Group

(a)
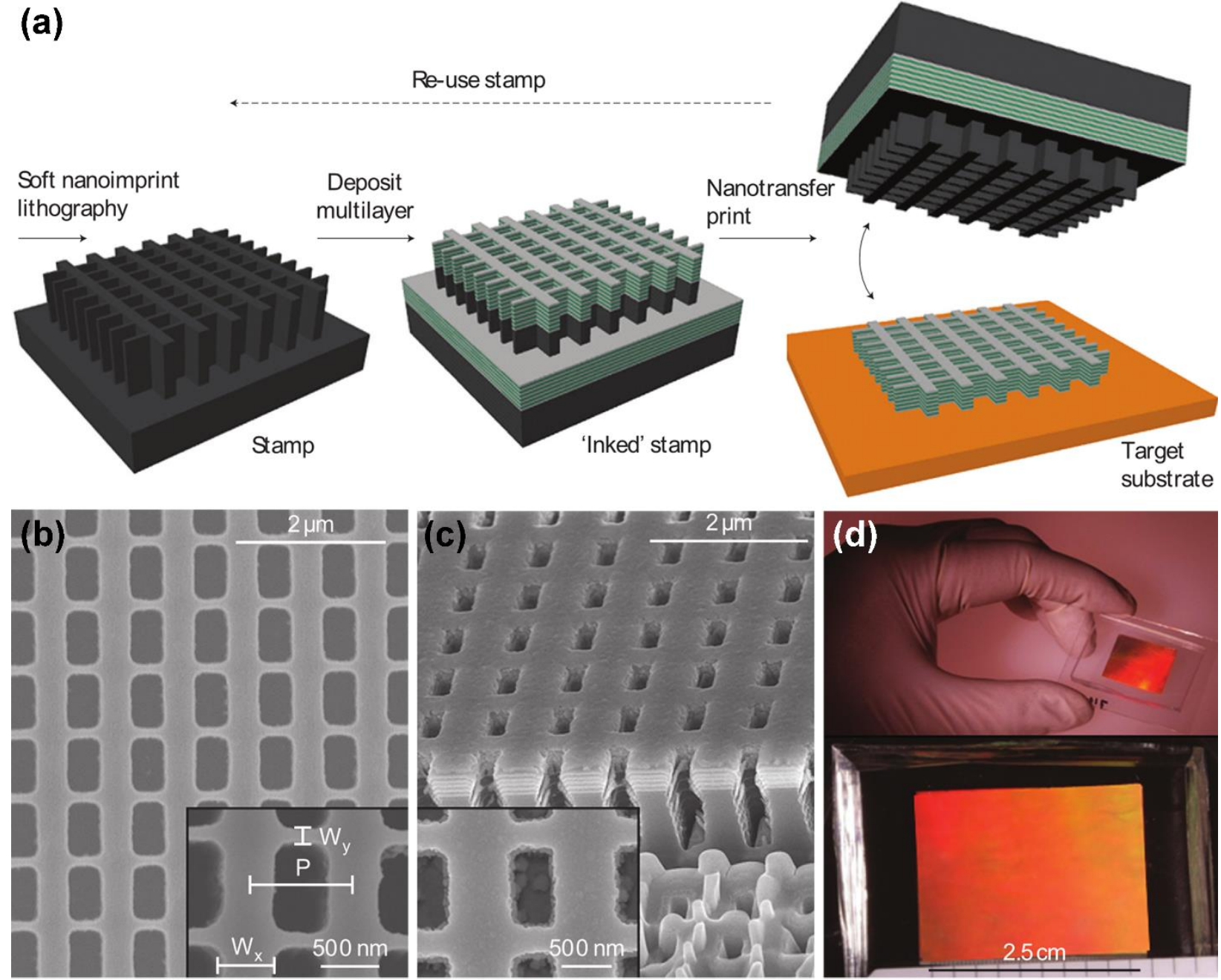

FIG. 8. (a) Schematic of a nanoimprint transfer technique used to define multilayered optical metamaterials, utilizing the process as shown in Fig. 4(a-f) with a high aspect ratio etched silicon master. (b,c) Scanning electron micrographs showing the etched silicon master defined by a Bosch process in (b) and an angled view post-deposition showing the multilayer film structure in (c). (d) The multilayered metamaterial transferred to a target substrate. Image reproduced with permission from Nat. Nanotechnol. 6, 402 (2011). Copyright

Nature

Publishing

Group

large writing times that typically accompany this method, any fluctuations in the current can give rise to inconsistent exposure, and result in variable developing times for PMMA as well as introduce geometrical errors.

Three-Dimensional Fabrication Techniques
Interest in fabricating three-dimensional (3D) terahertz and optical metamaterials. ${ }^{7,11,50,77,119,124}$ is driven by an increasing interest in applications that can provide the ability of imaging below diffraction limit, cloaking, quantum levitation, and sensing. Planar fabrication techniques 
have been largely employed in the synthesis of multilayered $3 \mathrm{D}$

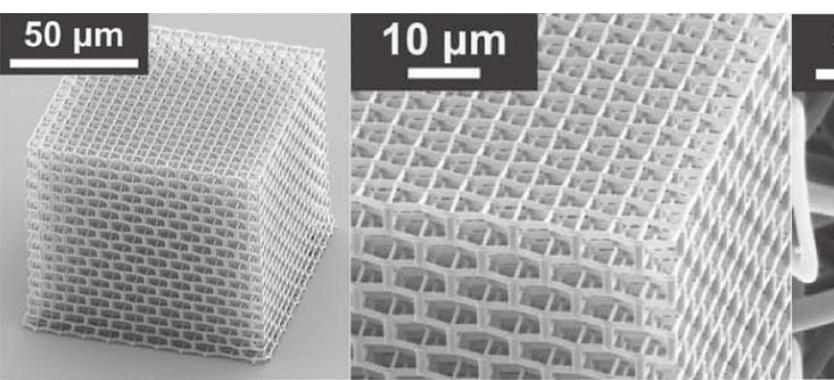

FIG. 9. Scanning electron micrographs of a 3D mechanical metamaterial showing three different magnifications of the complex structure defined using direct laser writing. Image reproduced with permission from Rapid Commun. 34, 335 (2013). Copyright 2013 Wiley-Blackwell

(a)
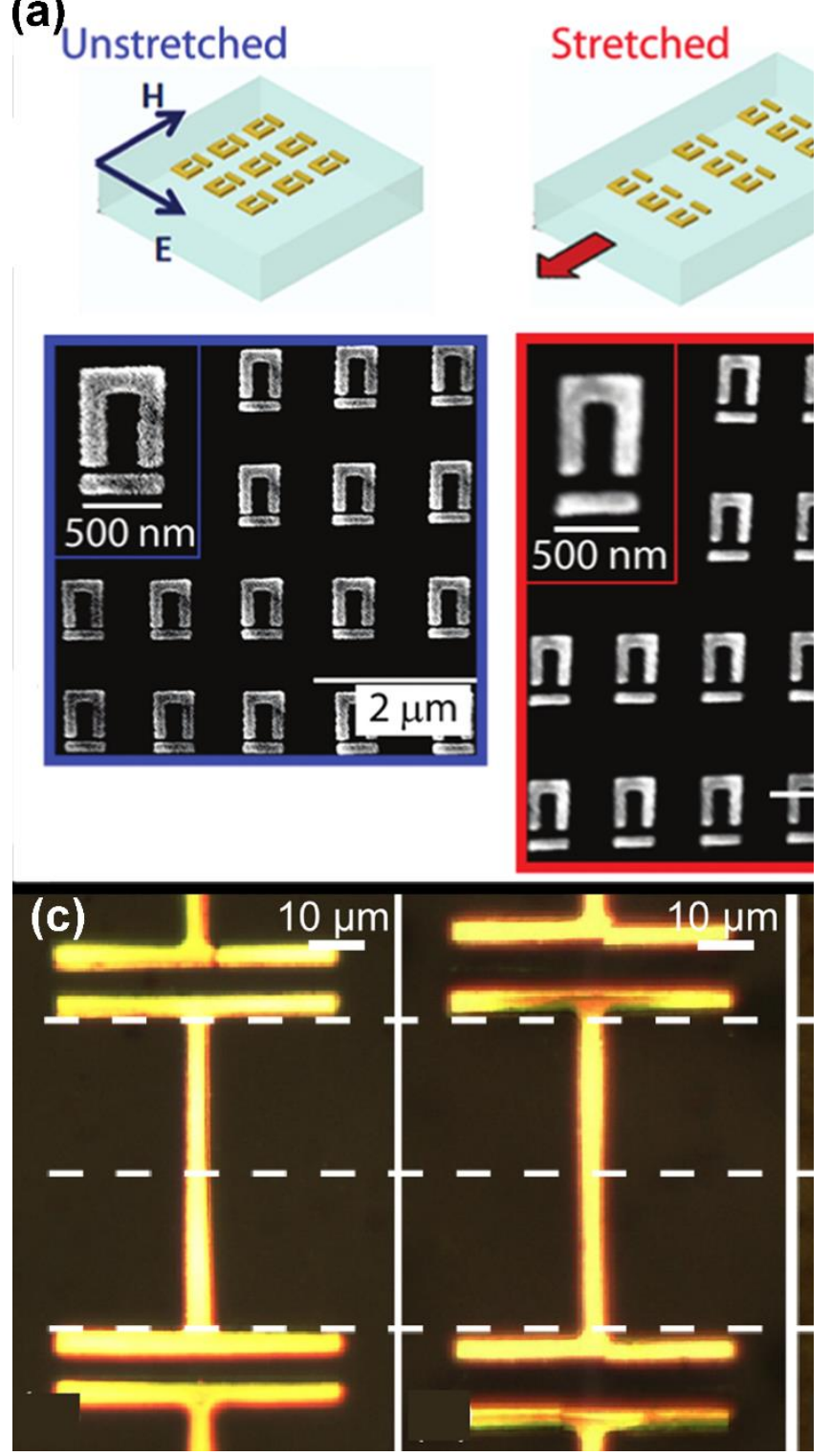

FIG. 10. Tuning metamaterials by mechanical deformation of elastomeric PDMS substrate. Mechanically tunable: (a) optical metamaterial, (b) honeycomb terahertz metamaterial, and (c) 'I'-shaped flat (two left-side panels) and interdigitated (two right-side panels) terahertz metamaterial. Images reproduced with permission from
Nano. Lett. 10, 4222 (2010). Copyright 2010 American Chemical Society, Nano. Lett. 10, 2783 (2010). Copyright 2010 American Chemical Society, reproduced with permission from Opt. Lett. 38, 2104 (2013). Copyright 2013 Optical Society of Americametamaterials owing to their simplicity. ${ }^{10,11,52,65,77,84,85,135}$ However, such multilayered metamaterials often suffer from anisotropic responses. Advanced applications such as cloaks require true isotropic metamaterials that can provide a spatial control on the permittivity $(\epsilon)$ and permeability $(\mu)$ over a defined volume. Metamaterials with isotropic negative $\epsilon$ and $\mu$ cannot be achieved via planar fabrication processes. Further advances in fabrication techniques for nonplanar metamaterials are therefore required to achieve sub-wavelength dimension metamaterials with isotropic responses.

Advanced fabrication techniques such as imprint lithography, ${ }^{10,}{ }^{52}$ microstereolithography, ${ }^{136}$ vertical pillar superlattice, ${ }^{137}$ multi-photon polymerization, ${ }^{7}$ multilayer electroplating (Figure 6), ${ }^{7}$ and interference lithography ${ }^{138}$ have been explored to demonstrate 3D metamaterial structures. However, such advanced 3D fabrication techniques are still limited by their fabrication complexity and ability for realization on or transfer onto flexible substrates.

A combination of laser writing and chemical vapor deposition techniques have also been explored to create a 3D split ring resonator (SRR) metamaterial. ${ }^{119}$ The chemical vapor deposition technique allows uniform coating of metal films around the structure, which cannot be achieved through physical vapor deposition techniques. 3D SRRs with varying heights have been experimentally shown to demonstrate resonance at different frequencies.

Focused ion beam (FIB) milling is another 3D fabrication technique used to realize nanometer size features with high aspect ratios. A fishnet resonator design made using the FIB technique was the one of the first 3D optical metamaterials realized with simultaneously negative $\epsilon$ and $\mu$ over a broad spectral range. ${ }^{50}$ This $3 \mathrm{D}$ fishnet structure was developed by multilayer deposition of metal and dielectric layers - 21 alternating layers of silver (11 layers) and magnesium fluoride (10 layers). Subsequently, FIB milling was used to etch nanometer size features with high aspect ratios (Figure 7)..$^{50}$

Using a similar patterning approach while using plasma etching, Chanda et al. reported a fishnet structure fabricated using nano-imprint lithography and multilayer electron beam evaporation techniques to demonstrate a high throughput negative index metamaterial. ${ }^{52}$ These fishnet structures were transferable onto an elastomeric PDMS substrate, which was subsequently transferred to other rigid substrates using a transfer and printing technique, as shown in Figure 8. The aforementioned nanofabrication technique can be used to design large-area 3D metamaterials operating at infrared and optical frequencies. In future, a combination of printing techniques with other large-area lithography techniques have the potential to generate sophisticated negative index metamaterials which are currently unavailable due to the limitations of 
patterning footprint, low aspect ratio structures, and low repeatability.

3D direct laser writing (DLW) fabrication technique can be used to develop complex geometry metamaterial structures. The DLW technique involves a tightly focused laser beam incident onto a diffraction-limited spot within the volume of the photoresist. This allows the creation of sub-micrometer scale features in three dimensions, that can be patterned to arbitrary shapes and complex interconnected networks which cannot be fabricated using conventional lithography techniques. Whilst, direct write techniques can achieve high resolution, they suffer from low throughput and are compatible only with a select range of substrates. The resolution can be further improved using multiple wavelength stimulation/depletion techniques. Recently, Wu et al. have established an alternative route to realizing high contrast, broadband circular-dichroic properties using an all metal, self-supported, freestanding chiral metafoil that can be manufactured by imprinting and hot embossing. Buckmann et al. recently demonstrated a modified "dip-in" DLW technique to obtain millimeter height mechanical metamaterial structures. ${ }^{121}$ The height that can be obtained using the standard DLW is usually limited to tens of micrometers. This limitation was overcome by the "dip-in" 3D DLW technique, wherein the photoresist itself acts as an immersion fluid between the substrate and the microscope lens. This enables the fabrication of structures with an overall height in the order of millimeters. Figure 9 shows a SEM micrograph of a typical 3D metamaterial synthesized using the DLW technique which was shown to exhibit mechanical tunability.

Membrane projection lithography (MPL) is another advanced fabrication technique capable of developing micrometer-scale 3D metamaterials. Using this technique, SRRs have been patterned directly on the face of a SU-8 substrate arranged in a cubical orientation. ${ }^{122}$ With such a high degree of control over the pattern and deposition of thin films, MPL has the potential to revolutionize future 3D infrared and optical metamaterial structures with complex geometries. A SRR design using the MPL technique was used to demonstrate the coupling of a magnetic field to an incident electromagnetic wave. ${ }^{122}$

Although the major techniques described above are promising for the creation of 3D metamaterial structures, they are still not sufficiently versatile. Some are complex and require multiple fabrication steps which degrade the resolution of the structures, while others are limited by the materials used for patterning as well as choice of suitable substrates. In addition, transfer techniques rely on surface chemistry making them substrate dependent. Although, complex fabrication techniques involving a fusion of soft lithography and photolithography have been used to for direct transfer of patterns onto highly curved substrates, achieving high resolution patterns $(<0.1 \mu \mathrm{m})$ still remains a challenge. ${ }^{139}$

\section{TUNING TECHNIQUES FOR METAMATERIALS}

Mechanical Tuning
Mechanically deformable flexible substrates are commonly used to tune the resonance frequency of metamaterials. $^{48,60,62,88,89,140-142}$ The application of a stretching force to the substrate alters the resonator geometry and also inter-resonator electric and magnetic couplings, which consequently change the resonance frequency of the resonators. ${ }^{88}$ Such an approach has been used to demonstrate dynamic tuning of Fano resonances by applying uniaxial mechanical stress to a plasmonic nanostructure on a PDMS membrane. ${ }^{60}$ The mechanical tuning of plasmonic nanostructures offers a new pathway towards the development of tunable nanophotonic devices such as tunable filters and sensors. Mechanical tuning is particularly effective for plasmonic nanostructures exhibiting coupled plasmon resonances, due to its high sensitivity to changes in structural parameters. ${ }^{35,51,60,62}$ It also allows control over the symmetry of a nanostructure or nanostructured unit cell, on which optical properties are critically dependent. ${ }^{60}$

The mechanical deformation of elastomeric substrates has been shown to modify the distance between the resonant elements in a controlled manner. ${ }^{18,48,51,60,88,140}$ Figure 10 shows examples of metamaterials fabricated on a PDMS substrate that have been used to demonstrate mechanical tuning of resonances at terahertz and optical frequencies. Figure 10(a) shows a SRR metamaterial designed to operate at optical frequencies, which is tuned by mechanical deformation of an elastomeric PDMS substrate. ${ }^{48}$ The resonators are controllably modified by 
(a)

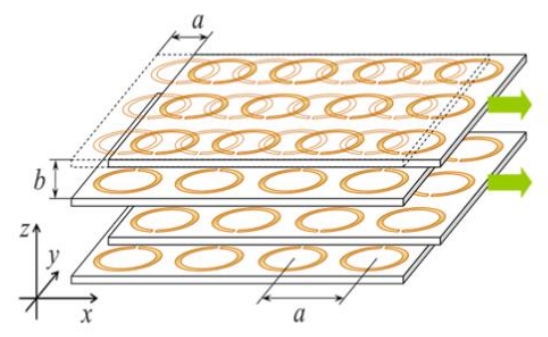

(b)

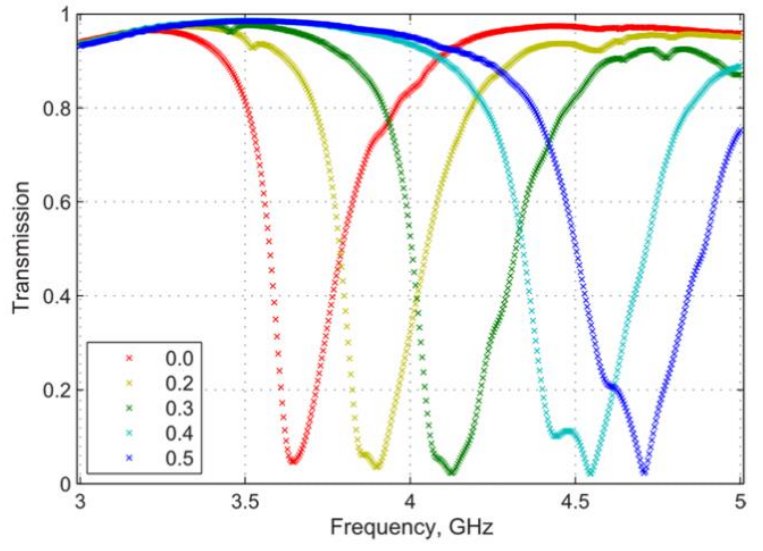

FIG. 11. Lattice displacement to create a reconfigurable metamaterial. (a) Schematic representation of shifting metasurfaces in relation to other layers. (b) Transmission response of the waveguide with different metamaterial slabs. Graphs with dips from right to left correspond to decreasing lattice shift. Image reproduced with permission from Appl. Phys. Lett. 95, 084105 (2009). Copyright 2009 American Institute of Physics

straining the PDMS substrate and demonstrate tunability larger than the resonant line width at optical frequencies. Experimental verification of the SRR structures demonstrated that relatively large uniaxial strains up to $50 \%$ caused shifts in the resonance frequency by $4 \%$. This tuning mechanism has also been implemented to demonstrate dynamic surface-enhanced infrared absorption, where the reflection signal is enhanced by a factor of $180 .{ }^{48}$

A plasmonic bow-tie antenna design on a PDMS substrate using nano-stencil lithography has also been demonstrated. ${ }^{51}$ The gap between the bow-tie antennas can induce unique electromagnetic responses such as plasmon-induced transparency and large near-field intensities. ${ }^{51}$ Mechanical deformation of these structures resulted in a red-shift in frequency as the gap was progressively reduced in steps of $10 \mathrm{~nm}$, from $45 \mathrm{~nm}$ to $25 \mathrm{~nm}$. Such surface-patterned metamaterials on PDMS can be wrapped around non-planar surfaces, such as optical fibers, and would possess the capability to enable novel functional photonic probes and antennas, that can be employed for monitoring environmental changes at remote locations.

Another example of a configuration used for mechanical tuning of metamaterials at terahertz frequencies is shown in Figure 10(b). ${ }^{136}$ It shows a wrinkled terahertz metamaterial on the surface of a pre-stretched PDMS substrate. The unit cells or meta-atoms created on the wrinkled configuration have a peak strain ratio of $52.1 \%$, which allows broadband transmission response and tuning of the metamaterials.

The honeycomb metamaterial design shown in Fig. 10(b) is symmetric in nature, and therefore, expected to have polarization independent responses. ${ }^{136}$ However, in a wrinkled state, the electromagnetic responses were observed to be polarization sensitive. It was shown that the transmission response for a transverse electric (TE) polarization was $90 \%$ stronger than the transverse magnetic (TM) counterpart. Such polarization-dependent properties of wrinkled metamaterials find application in reconfigurable phase retarders. ${ }^{140}$

Uniaxial 'I'-shaped resonators [Fig. 10(c)], with and without interdigitated gaps, fabricated on PDMS represent another design demonstrated for mechanical tuning of the resonance frequency in the terahertz regime. Strain of $10 \%$ were shown to enable over $8 \%$ tuning of the resonance frequency with a high degree of reversibility ${ }^{87}$ This compares with the $50 \%$ strain inducing just a $4 \%$ change in resonance frequency for the example in Fig. 10(a). ${ }^{48}$ The work with the uniaxial 'I'-shaped resonators was extended to designs with a four-fold symmetry in the form of a cross structure, and used to demonstrate polarization-dependent tuning and with potential applications in strain sensing. ${ }^{18}$

Although other methods for the manipulation of resonant components have been employed, and will be discussed in the next section, mechanical deformation offers higher control over the overall design, symmetry, and the behavior of the system without altering its components. $^{48}$

Apart from mechanical tuning, a wide range of other techniques have also been explored. These include reconfigurable metamaterials via electromechanical displacements, ${ }^{143}$ lattice displacement, ${ }^{144}$ thermal annealing, ${ }^{89}$ and change in superfluid density. ${ }^{145}$ Incorporation of phase-change media, semiconductors, graphene, carbon nanotubes, nonlinear-components, and liquid crystals with metamaterials are also strategies that have been studied and have been thoroughly discussed in another review article by Zheludev et al. ${ }^{68}$ Some of the other commonly studied tuning techniques are briefly described below.

\section{Electromechanical Displacements}

Extending the concept of using mechanical deformation for tuning, the application of an electrical stimulus to induce mechanical strain leads to a category of electromechanical tuning. In electromechanical tuning, an applied external bias voltage induces mechanical deformation of resonator structures. Lin et al. fabricated an array of double split-ring resonators (DSRRs) suspended in air in the form of cantilever structures utilized in microelectromechanical systems (MEMS) design. ${ }^{143}$ The cantilever structure displaces on excitation by an external bias voltage and subsequently returns to its original location on removal of the bias due to the stress induced in the cantilever. This electromechanical tuning of DSRRs was used to demonstrate tunable filters operating at terahertz frequencies. ${ }^{143}$ Here, the electrostatic 
force applied as a bias voltage controls the degree of bending in the cantilevers. A bias voltage of $20 \mathrm{~V}$ enabled a resonant frequency tuning range of $0.5 \mathrm{THz}$.

\section{Other Tuning Techniques}

The tuning techniques discussed in this section have been demonstrated for tuning metamaterials fabricated on various rigid platforms. These techniques can be adapted for achieving tunability in flexible metamaterials. The degree of tunability is expected to increase for techniques such as lattice displacement andthermal stimulation, as the flexibility provides more degree of freedom for large manipulations due to higher intrinsic susceptibility to external stimulus.

\section{Lattice Displacement}

Figure 11(a) shows a schematic representation of reconfigurable metamaterials, where an adjustment to the lattice structure has been proposed by Lapine et al. for tuning the transmission characteristics of metamaterials. ${ }^{144}$ They exploited the resonant frequency dependence on the lattice parameters ( $a$ and $b$ ) and altered the periodic lateral displacement of the layers in the $x y$ plane. The lateral displacement of the metamaterial layers leads to a shift in the alignment of resonators in the $x$ and/or $y$ directions, resulting in dramatic alterations of resonance frequency as shown in Fig. 11(b). Using this approach, continuous tuning of resonance frequency was experimentally demonstrated This tuning technique can be further extended to a wide range of electromagnetic frequencies and alternative resonator geometries. To realize such smart continuous tuning structures at higher frequencies, micro- and nano-fabrication techniques that can generate large-area multilayer metamaterials are required, along with materials that enable good control over lattice displacements in all three directions.

\section{Thermal Stimulation}

Temperature control of permittivity has been demonstrated to tune the resonant frequency in the terahertz spectral regime. ${ }^{146}$ This relies on utilizing oxide materials with multifunctional properties, including susceptibility to temperature. A temperature-dependent nonmagnetic rod made of $\mathrm{SrTiO}_{3}$ (STO) has been used to demonstrate magnetic permeability tuning at terahertz frequencies by altering the temperature. Resonance frequency tunability of $\sim 44 \%$ was seen as the temperature was decreased from $300 \mathrm{~K}$ to $120 \mathrm{~K}$.

Reconfigurable metamaterials that reorient in response to a thermal stimulus have also been used to tune metamaterials. ${ }^{89}$ A SRR metamaterial design supported by a cantilever beam that bends out of its plane in response to thermal annealing has been demonstrated to tune the electromagnetic response. However, the thermal annealing actuation process suffers from a passive tuning effect, where the SRR once reoriented never returns to its initial state. Hence, active tuning mechanisms such as resistive, piezoelectric, and electrostatic actuation must be further explored to tune the metamaterial responses.

\section{CONCLUSIONS AND FUTURE OUTLOOK}

In this review, recent progress on metamaterials realized on flexible and elastomeric substrates are presented. This is covered by considering materials utilized in the realization of devices, state-of-the-art fabrication approaches, and techniques for tuning metamaterials resonances on flexible substrates (or in some cases techniques that can be extended to flexible devices).

Metamaterials have predominantly been realized on a wide variety of polymers, with the properties of the polymers playing a crucial role in functionality. This work looks at properties of conventional polymers in detail, and summarizes these in detail for their use by researchers. The importance of substrates with low electrical losses and excellent mechanical properties to create tunable metamaterials have been highlighted.

Fabrication techniques to realize metamaterials on flexible substrates have been reviewed. Conventional microfabrication and nanofabrication techniques have been utilized widely to realize terahertz and optical devices with an increasing shift to new techniques. Alternate fabrication techniques such as shadow mask lithography and nanoimprint lithography have also been explored to overcome some of the fabrication challenges posed by flexible substrates. Other complex fabrication techniques such as membrane projection lithography and direct laser writing techniques have the potential to enable creation of complex metamaterial structures with isotropic responses.

The mechanical deformation tuning is a promising approach to make metamaterials tunable and to obtain adaptive response without adding any further complexity to the design and fabrication of the structures. This technique allows dynamic tuning of metamaterials without the need for bias voltages, nonlinear components and MEMS switches. Also, mechanical tuning of metamaterials is not limited by the frequency of operation and it can be extended from microwave, ${ }^{19}$ terahertz, ${ }^{47,65,89}$ to optical frequencies. ${ }^{10,35,48,52}$ The ability to mechanically tune metamaterials has provided new degree of freedom in sensing at molecular levels by enhanced reflection signal of a dynamic surface infrared absorption. ${ }^{38,} 48,147$ Metamaterials and plasmonic nanostructures on flexible substrates are highly sensitive to structural parameters, which opens up new possibilities for photonic circuits, biological systems, antennas, sensors (strain, temperature, dielectric, biomolecular, chemical, etc.), energy harvesters, tunable cloaking devices operating over wide frequencies that can overcome the current limitations of specific wavelength and angle operation, and super-lenses to image at near atomic scales. Towards such applications, further research is being carried out to develop devices with enhanced resolution of the resonator structures to operate at higher frequencies, large-area flexible structures, 3D metamaterials, isotropic response structures, and fast tuning capabilities of mechanically deformable metamaterials.

The limitations of metamaterials have been overcome by making devices on flexible and elastomeric substrates to achieve tunable broadband responses. Fasci- 
nating work has been carried out in the field of terahertz metamaterials so far, to demonstrate antennas, filters, absorbers and sensors. This was mainly driven due to the lack of photonic components required to control/manipulate the terahertz radiation. Further research is being carried out to realize 3D terahertz metamaterials to fashion exotic applications. This will enable isotropic response metamaterials, which opens up a myriad of applications in terahertz, infrared and visible frequencies. The advancement of tuning mechanisms and continuous improvements in fabrication techniques are vital for the future of metamaterials.

Metamaterial antennas have found their way to commercial market in the form of lightweight and highly efficient antennas for routers, mobile phones and airport scanners. However, integrating the antennas with rest of the components has posed challenges, which can be overcome by creating metamaterials on thin flexible substrate. Metamaterials have also been used to harvest electromagnetic energy due to their natural ability to couple with the incident radiation and convert it from one form to another. ${ }^{46}$ Although, such energy harvesting has been demonstrated at microwave frequencies, it would require high-resolution large-area nanostructures to harvest energy when operating at higher frequencies. The future of metamaterials range from simple wireless charging devices to windows that are able to harvest energy previously considered impossible.

It can be concluded that flexible polymers offer advantages in tuning by mechanical deformation. Metamaterials on flexible substrates promise the potential of integration with other components and non-planar structures. The advancement of fabrication capabilities to obtain high-resolution micro- and nano-scale patterns on elastomeric substrates holds the key to the future of metamaterials.

\section{ACKNOWLEDGMENT}

Funding from the Australian Research Council through Discovery Projects DP1092717 (MB), DP1095151 (WW), DP110100262 (SS), and DP130100062 (SW, SS) is acknowledged. SS acknowledges Victoria and AFAS-Vic Fellowship support. The authors acknowledge collaborators and colleagues who contributed to work covered by this review and to engaging discussions.

\section{REFERENCES}

1 D. Smith, J. Pendry and M. Wiltshire, Science 305, 788 (2004).

2 H.-T. Chen, J. F. O'Hara, A. K. Azad, A. J. Taylor, R. D. Averitt, D. B. Shrekenhamer and W. J. Padilla, Nature Photon. 2, 295 (2008).

3 W. C. Chen, A. Totachawattana, K. Fan, J. L. Ponsetto, A. C. Strikwerda, X. Zhang, R. D. Averitt and W. J. Padilla, Phys. Rev. B. 85 (2012).

4 M. Choi, S. H. Lee, Y. Kim, S. B. Kang, J. Shin, M. H. Kwak, K. Y. Kang, Y. H. Lee, N. Park and B. Min, Nature 470, 369 (2011).

5 M. J. Dicken, K. Aydin, I. M. Pryce, L. A. Sweatlock, E. M. Boyd, S. Walavalkar, J. Ma and H. A. Atwater, Opt. Express 17, 18330 (2009).
6 E. Ekmekci, A. C. Strikwerda, K. Fan, G. Keiser, X. Zhang, G. Turhan-Sayan and R. D. Averitt, Phys. Rev. B. 83, 193103 (2011).

7 K. B. Fan, A. C. Strikwerda, H. Tao, X. Zhang and R. D. Averitt, Opt. Express 19, 12619 (2011).

8 S. Iyer, S. Popov and A. T. Friberg, Appl. Opt. 50, 3958 (2011).

9 R. C. McPhedran, I. V. Shadrivov, B. T. Kuhlmey and Y. S. Kivshar, NPG. Asia. Mater. 3, 100 (2011).

10 I. Bergmair, B. Dastmalchi, M. Bergmair, A. Saeed, W. Hilber, G. Hesser, C. Helgert, E. Pshenay-Severin, T. Pertsch, E. B. Kley, U. Hubner, N. H. Shen, R. Penciu, M. Kafesaki, C. M. Soukoulis, K. Hingerl, M. Muehlberger and R. Schoeftner, Nanotechnology 22, 325301 (2011).

11 N. Liu, H. Guo, L. Fu, S. Kaiser, H. Schweizer and H. Giessen, Nat. Mater. 7, 31 (2008).

12 H. O. Moser and C. Rockstuhl, Laser. Photon. Rev. 6, 219 (2012).

13 C. M. Soukoulis and M. Wegener, Nature Photon. 5, 523 (2011).

14 J. B. Pendry, D. Schurig, J. J. Mock, B. J. Justice, S. A. Cummer, A. F. Starr and D. R. Smith, Science 314, 977 (2006).

15 S. Aksu, M. Huang, A. Artar, A. A. Yanik, S. Selvarasah, M. R. Dokmeci and H. Altug, Adv Mater 23 (2011).

16 T. Chen, S. Li and H. Sun, Sensors (Basel) 12, 2742 (2012).

17 J. Kim, H. Son, D. J. Cho, B. Geng, W. Regan, S. Shi, K. Kim, A. Zettl, Y. R. Shen and F. Wang, Nano Lett. 12, 5598 (2012).

18 J. Li, C. M. Shah, W. Withayachumnankul, B. S. Ung, A. Mitchell, S. Sriram, M. Bhaskaran, S. Chang and D. Abbott, Opt. Lett. 38, 2104 (2013).

19 R. Melik, E. Unal, N. K. Perkgoz, C. Puttlitz and H. V. Demir, Appl. Phys. Lett. 95, 181105 (2009).

20 B. Ng, S. M. Hanham, V. Giannini, Z. C. Chen, M. Tang, Y. F. Liew, N. Klein, M. H. Hong and S. A. Maier, Opt. Express 19, 14653 (2011).

21 H. Tao, L. R. Chieffo, M. A. Brenckle, S. M. Siebert, M. K. Liu, A. C. Strikwerda, K. B. Fan, D. L. Kaplan, X. Zhang, R. D. Averitt and F. C. Omenetto, Adv. Mater. 23, 3197 (2011).

22 X. Xu, B. Peng, D. Li, J. Zhang, L. M. Wong, Q. Zhang, S. Wang and Q. Xiong, Nano Lett. 11, 3232 (2011).

23 J. B. Pendry, Contemp. Phys. 45, 191 (2004).

24 N. Fang, H. Lee, C. Sun and X. Zhang, Science 308, 534 (2005).

25 Z. Jacob, L. V. Alekseyev and E. Narimanov, Opt. Express 14, 8247 (2006).

26 Z. Liu, H. Lee, Y. Xiong, C. Sun and X. Zhang, Science 315, 1686 (2007).

27 C. Y. Luo, S. G. Johnson, J. D. Joannopoulos and J. B. Pendry, Phys. Rev. B. 68, 045115 (2003).

28 V. M. Shalaev, Nature Photon. 1, 41 (2007).

29 X. Zhang and Z. Liu, Nat. Mater. 7, 435 (2008).

30 M. Choi, J.-H. Choe, B. Kang and C.-G. Choi, Curr. Appl. Phys. 13, 1723 (2013).

31 S. Lee, L. Li, Z. Wang, W. Guo, Y. Yan and T. Wang, Appl. Opt. 52, 7265 (2013).

32 F. Xu, G. Chen, C. Wang, B. Cao and Y. Lou, Opt. Lett. 38, 3819 (2013).

33 S. Zhang, S. Liu, X. Yang, C. Wang and X. Luo, J. Nanophotonics. 7, 073080 (2013).

34 L. Huang, X. Chen, H. Muehlenbernd, H. Zhang, S. Chen, B. Bai, Q. Tan, G. Jin, K.-W. Cheah, C.-W. Qiu, J. Li, T. Zentgraf and S. Zhang, Nat. Commun. 4, 2808 (2013).

35 R. Caputo, U. Cataldi, A. Cunningham, L. De Sio, T. Bürgi and C. Umeton, Asia Commun. Photonics Conf. Exhib., ACPC.2012 PAF4D.8 (2012).

36 Y.-C. Chang, S. Yin, C. Wang and C. Luo, Proc. SPIE, 8120 $81201 \mathrm{M}(2011)$.

37 J. Y. Ou, E. Plum, L. Jiang and N. I. Zheludev, Nano Lett. 11, 2142 (2011). 
38 J. Y. Ou, E. Plum, J. Zhang and N. I. Zheludev, Nat. Nanotechnol. 8, 252 (2013).

39 T.-J. Yen, D. Wu, N. Fang and X. Zhang, in Optical Science and Technology, the SPIE 49th Annual Meeting (International Society for Optics and Photonics, 2004), pp. 100-106.

40 C. L. Yu, H. Kim, N. de Leon, I. W. Frank, J. T. Robinson, M. McCutcheon, M. Liu, M. D. Lukin, M. Loncar and H. Park, Nano Lett. 13, 248 (2013).

41 Z. H. Jiang, S. Yun, F. Toor, D. H. Werner and T. S. Mayer, ACS Nano 5, 4641 (2011).

42 D. Y. Shchegolkov, A. K. Azad, J. F. O'Hara and E. I. Simakov, Phys. Rev. B. 82, 205117 (2010).

43 C. M. Watts, X. Liu and W. J. Padilla, Adv. Mater. 24, OP98 (2012).

44 Q.-Y. Wen, H.-W. Zhang, Q.-H. Yang, Z. Chen, B.-H. Zhao, Y. Long and Y.-L. Jing, edited by D. X.-Y. Jiang (InTech, 2012).

45 O. M. Ramahi, T. S. Almoneef, M. AlShareef and M. S. Boybay, Appl. Phys. Lett. 101, 173903 (2012).

46 A. M. Hawkes, A. R. Katko and S. A. Cummer, Appl. Phys. Lett. 103, 163901 (2013).

47 H. Tao, N. Landy, K. Fan, A. Strikwerda, W. Padilla, R. Averitt and X. Zhang, in Electron Devices Meeting, 2008. IEDM 2008. IEEE International (IEEE, 2008), pp. 1-4.

48 I. M. Pryce, K. Aydin, Y. A. Kelaita, R. M. Briggs and H. A. Atwater, Nano Lett. 10, 4222 (2010).

49 W. J. Padilla, D. N. Basov and D. R. Smith, Mater. Today 9 , 28 (2006)

50 J. Valentine, S. Zhang, T. Zentgraf, E. Ulin-Avila, D. A. Genov, G. Bartal and X. Zhang, Nature 455, 376 (2008).

51 S. Aksu, M. Huang, A. Artar, A. A. Yanik, S. Selvarasah, M. R. Dokmeci and H. Altug, Adv. Mater. 23, 4422 (2011).

52 D. Chanda, K. Shigeta, S. Gupta, T. Cain, A. Carlson, A. Mihi, A. J. Baca, G. R. Bogart, P. Braun and J. A. Rogers, Nat. Nanotechnol. 6, 402 (2011).

53 I. E. Khodasevych, C. M. Shah, S. Sriram, M. Bhaskaran, W. Withayachumnankul, B. S. Y. Ung, H. Lin, W. S. T. Rowe, D. Abbott and A. Mitchell, Appl. Phys. Lett. 100, 061101 (2012).

54 N. Tiercelin, P. Coquet, R. Sauleau, V. Senez and H. Fujita, J. Micromech. Microeng. 16, 2389 (2006).

55 X. Liu, S. MacNaughton, D. B. Shrekenhamer, H. Tao, S. Selvarasah, A. Totachawattana, R. D. Averitt, M. R. Dokmeci, S. Sonkusale and W. J. Padilla, Appl. Phys. Lett. 96, 011906 (2010).

56 J. A. Rogers and R. G. Nuzzo, Mater. Today 8, 50 (2005).

57 J. A. Rogers, T. Someya and Y. Huang, Science 327, 1603 (2010).

58 K. Iwaszczuk, A. C. Strikwerda, K. B. Fan, X. Zhang, R. D. Averitt and P. U. Jepsen, Opt. Express 20, 635 (2012).

59 H. C. Ko, G. Shin, S. Wang, M. P. Stoykovich, J. W. Lee, D. H. Kim, J. S. Ha, Y. Huang, K. C. Hwang and J. A. Rogers, Small 5, 2703 (2009).

60 Y. Cui, J. Zhou, V. A. Tamma and W. Park, ACS Nano 6, 2385 (2012).

61 J. Henzie, M. H. Lee and T. W. Odom, Nat. Nanotechnol. 2, 549 (2007).

62 V. A. Tamma, Y. Cui, J. Zhou and W. Park, in Quantum Electronics and Laser Science Conference (Optical Society of America, 2012), pp. QW1B.6.

63 L. J. Liang, J. Q. Yao and X. Yan, Chin. Phys. Lett. 29, 094209 (2012).

64 P. K. Singh, K. A. Korolev, M. N. Afsar and S. Sonkusale, Appl. Phys. Lett. 99, 264101 (2011).

65 H. Tao, C. M. Bingham, A. C. Strikwerda, D. Pilon, D. Shrekenhamer, N. I. Landy, K. Fan, X. Zhang, W. J. Padilla and R. D. Averitt, Phys. Rev. B. 78, 241103 (2008).

66 A. Q. Liu, W. M. Zhu, D. P. Tsai and N. I. Zheludev, J. Opt. 14, 114009 (2012).

67 N. I. Zheludev, Opt. Photonics. News. 22, 30 (2011).

68 N. I. Zheludev and Y. S. Kivshar, Nat. Mater. 11, 917 (2012).
69 H. Y. Hwang, N. C. Brandt, K. Fan, X. Zhang, R. D. Averitt and $\mathrm{K}$. Nelson, in International Conference on Ultrafast Phenomena (Optical Society of America, 2014), pp. P3. 43.

70 R. Ortuño, C. García-Meca and A. Martínez, Plasmonics 9, 1143 (2014).

71 R. Singh and N. Zheludev, Nature Photon. 8, 679 (2014).

72 I. Al-Naib, G. Sharma, M. M. Dignam, H. Hafez, A. Ibrahim, D. G. Cooke, T. Ozaki and R. Morandotti, in CLEO: Science and Innovations (Optical Society of America, 2013), Vol. CLEO_SI.2013,pp. CM4J. 7.

73 K. Fan, A. C. Strikwerda, X. Zhang and R. D. Averitt, Phys. Rev. B. 87, 161104 (2013).

74 N. K. Grady, J. E. Heyes, D. R. Chowdhury, Y. Zeng, M. T. Reiten, A. K. Azad, A. J. Taylor, D. A. Dalvit and H.-T. Chen, Science 340, 1304 (2013).

75 J. P. Turpin, J. A. Bossard, K. L. Morgan, D. H. Werner and P. L. Werner, Int. J. Antennas. Propag. 2014, 429837 (2014).

76 N. K. Grady, B. G. Perkins Jr, H. Y. Hwang, N. C. Brandt, D. Torchinsky, R. Singh, L. Yan, D. Trugman, S. A. Trugman and Q. Jia, New. J. Phys. 15, 105016 (2013).

77 A. K. Azad, H. T. Chen, X. Lu, J. Gu, N. R. Weisse-Bernstein, E. Akhadov, A. J. Taylor, W. Zhang and J. F. O'Hara, IEEE Trans Terahertz Sci Technol. 2, 15 (2009).

78 Z. C. Chen, N. R. Han, Z. Y. Pan, Y. D. Gong, T. C. Chong and M. H. Hong, Opt. Mater. Express. 1, 151 (2011).

79 A. Di Falco, M. Ploschner and T. F. Krauss, New. J. Phys. 12, 113006 (2010).

80 N. R. Han, Z. C. Chen, C. S. Lim, B. Ng and M. H. Hong, Opt. Express 19, 6990 (2011).

81 Y. L. Hor, Z. Szabo, H. C. Lim, J. F. Federici and E. P. Li, Appl. Opt. 49, 1179 (2010).

82 G. Kenanakis, R. Zhao, A. Stavrinidis, G. Konstantinidis, N. Katsarakis, M. Kafesaki, C. M. Soukoulis and E. N. Economou, Opt. Mater. Express. 2, 1702 (2012).

83 Y. Li, L. W. Tan, X. T. Hao, K. S. Ong, F. Zhu and L. S. Hung, Appl. Phys. Lett. 86, 153508 (2005).

84 F. Miyamaru, M. W. Takeda and K. Taima, Appl. Phys. Express. 2, 042001 (2009).

85 H. Tao, A. C. Strikwerda, K. Fan, C. M. Bingham, W. J. Padilla, X. Zhang and R. D. Averitt, J. Phys. D: Appl. Phys. 41, 232004 (2008)

86 L. Liu, W.-c. Chen, D. A. Powell, W. J. Padilla, F. Karouta, H. T. Hattori, D. N. Neshev and I. V. Shadrivov, Appl. Phys. Lett. 105, 151102 (2014).

87 W. Park and J.-B. Lee, Appl. Phys. Lett. 85, 4845 (2004)

88 J. Li, C. M. Shah, W. Withayachumnankul, B. S. Y. Ung, A. Mitchell, S. Sriram, M. Bhaskaran, S. Chang and D. Abbott, Appl. Phys. Lett. 102, 121101 (2013).

89 H. Tao, A. C. Strikwerda, K. Fan, W. J. Padilla, X. Zhang and R. D. Averitt, Phys. Rev. Lett. 103, 147401 (2009).

90 T. Tumkur, G. Zhu, P. Black, Y. A. Barnakov, C. E. Bonner and M. A. Noginov, Appl. Phys. Lett. 99, 151115 (2011).

91 N. Gibbons, J. J. Baumberg, C. L. Bower, M. Kolle and U. Steiner, Adv. Mater. 21, 3933 (2009).

92 R. R. A. Syms, L. Solymar, I. R. Young and T. Floume, J. Phys. D: Appl. Phys. 43, 055102 (2010).

93 I. W. Moran, A. L. Briseno, S. Loser and K. R. Carter, Chem. Mater. 20, 4595 (2008).

94 J. Lötters, W. Olthuis, P. Veltink and P. Bergveld, J. Micromech. Microeng. 7, 145 (1997).

95 W. A. MacDonald, J. Mater. Chem. 14, 4 (2004)

96 M.-C. Choi, Y. Kim and C.-S. Ha, Prog. Polym. Sci. 33, 581 (2008).

97 A. Thompson and D. Woods, Trans. Faraday Society 52, 1383 (1956).

98 S. Costanzo, A. Borgia, I. Venneri and G. D. Massa, Radio. Eng. 20, 785 (2011).

99 Y.-S. Jin, G.-J. Kim and S.-G. Jeon, J. Korean. Phys. Soc. 49 513 (2006) 
100 P. Gutruf, C. M Shah, S. Walia, H. Nili, A. S Zoolfakar, C. Karnutsch, K. Kalantar-zadeh, S. Sriram and M. Bhaskaran, NPG. Asia. Mater. 5, e62 (2013).

101 T. Buma, M. Spisar and M. O’Donnell, Appl. Phys. Lett. 79, 548 (2001).

102 N. Bowden, S. Brittain, A. G. Evans, J. W. Hutchinson and G. M. Whitesides, Nature 393, 146 (1998).

103 J. L. Fritz and M. J. Owen, J. Adhesion. 54, 33 (1995).

104 S. P. Lacour, S. Wagner, Z. Huang and Z. Suo, Appl. Phys. Lett. 82, 2404 (2003).

105 R. C. Pangule, I. Banerjee and A. Sharma, J. Chem. Phys. 128, 234708 (2008).

106 F. C. Krebs, Sol. Energy Mater. Sol. Cells 93, 1636 (2009).

107 T. Li, Z. Huang, Z. Xi, S. P. Lacour, S. Wagner and Z. Suo, Mech. Mater. 37, 261 (2005).

108 N. Lu, X. Wang, Z. Suo and J. Vlassak, Appl. Phys. Lett. 91, 221909 (2007).

109 K. C. Cheung, P. Renaud, H. Tanila and K. Djupsund, Biosens. Bioelectron. 22, 1783 (2007).

110 M. Zirkl, A. Haase, A. Fian, H. Schön, C. Sommer, G. Jakopic, G. Leising, B. Stadlober, I. Graz, N. Gaar, R. Schwödiauer, S. Bauer-Gogonea and S. Bauer, Adv. Mater. 19, 2241 (2007).

111 K. Nomura, H. Ohta, A. Takagi, T. Kamiya, M. Hirano and H. Hosono, Nature 432, 488 (2004).

112 C. Yan, J. H. Cho and J.-H. Ahn, Nanoscale 4, 4870 (2012).

113 W. Reddish, Trans. Faraday Society 46, 459 (1950).

114 P. Zoller and P. Bolli, J. Macromol. Sci., Phys. B18, 555 (1980).

115 G. X. Li, S. M. Chen, W. H. Wong, E. Y. B. Pun and K. W. Cheah, Opt. Express 20, 397 (2012).

116 C. M. Shah, S. Sriram, M. Bhaskaran, M. Nasabi, T. G. Nguyen, W. S. T. Rowe and A. Mitchell, J. Microelectromech. Syst. 21, 1410 (2012).

117 G. M. Kim, M. A. F. van den Boogaart and J. Brugger, Microelectron. Eng. 67-68, 609 (2003)

118 A. S. Quick, J. Fischer, B. Richter, T. Pauloehrl, V. Trouillet, M. Wegener and C. Barner-Kowollik, Macromol. Rapid Commun. 34, 335 (2013).

119 M. S. Rill, C. Plet, M. Thiel, I. Staude, G. von Freymann, S. Linden and M. Wegener, Nat. Mater. 7, 543 (2008).

120 K. Fan, A. C. Strikwerda, X. Zhang and R. D. Averitt, arXiv preprint arXiv:1301.3977 (2013).

121 T. Buckmann, N. Stenger, M. Kadic, J. Kaschke, A. Frolich, T. Kennerknecht, C. Eberl, M. Thiel and M. Wegener, Adv. Mater. 24, 2710 (2012).

122 D. B. Burckel, J. R. Wendt, G. A. Ten Eyck, J. C. Ginn, A. R. Ellis, I. Brener and M. B. Sinclair, Adv. Mater. 22, 5053 (2010).

123 S. Salvatore, A. Demetriadou, S. Vignolini, S. S. Oh, S. Wuestner, N. A. Yufa, M. Stefik, U. Wiesner, J. J. Baumberg and O. Hess, Adv. Mater. 25, 2713 (2013).

124 D. B. Shao and S. C. Chen, Nano Lett. 6, 2279 (2006).

125 B. M. Durnin, C. M. Shah, S. Sriram and M. Bhaskaran, Proc. SPIE, 8204820437 (2011).

126 M. Apanius, P. B. Kaul and A. R. Abramson, Sensor Actuat. A.- Phys. 140, 168 (2007).

127 S. C. Nemat-Nasser, A. V. Amirkhizi, W. J. Padilla, D. N. Basov, S. Nemat-Nasser, D. Bruzewicz and G. Whitesides, Phys. Rev. E. 75, 036614 (2007).

128 H. Tao, J. J. Amsden, A. C. Strikwerda, K. B. Fan, D. L. Kaplan, X. Zhang, R. D. Averitt and F. G. Omenetto, Adv. Mater. 22, 3527 (2010).

129 Y. Qin, A. Pan, L. Liu, O. Moutanabbir, R. B. Yang and M. Knez, ACS Nano 5, 788 (2011).

130 Y. Xia and G. M. Whitesides, Annu. Rev. Mater. Sci. 28, 153 (1998).

131 D. Qin, Y. Xia and G. M. Whitesides, Nat. Protoc. 5, 491 (2010).
132 Y. Xia, E. Kim, X.-M. Zhao, J. A. Rogers, M. Prentiss and G. M. Whitesides, Science 273, 347 (1996).

133 M. A. Meitl, Z.-T. Zhu, V. Kumar, K. J. Lee, X. Feng, Y. Y. Huang, I. Adesida, R. G. Nuzzo and J. A. Rogers, Nat. Mater. 5, 33 (2005).

134 M. H. Lee, M. D. Huntington, W. Zhou, J.-C. Yang and T. W. Odom, Nano Lett. 11, 311 (2010).

135 X. G. Peralta, M. C. Wanke, C. L. Arrington, J. D. Williams, I. Brener, A. Strikwerda, R. D. Averitt, W. J. Padilla, E. Smirnova, A. J. Taylor and J. F. O'Hara, Appl. Phys. Lett. 94, 161113 (2009).

136 D. Wu, N. Fang, C. Sun, X. Zhang, W. J. Padilla, D. N. Basov, D. R. Smith and S. Schultz, Appl. Phys. Lett. 83, 201 (2003).

137 J. M. Lee, J. W. Choung, J. Yi, D. H. Lee, M. Samal, D. K. Yi, C. H. Lee, G. C. Yi, U. Paik, J. A. Rogers and W. I. Park, Nano Lett. 10, 2783 (2010).

138 S. Zhang, W. Fan, B. Minhas, A. Frauenglass, K. Malloy and S. Brueck, Phys. Rev. Lett. 94, 037402 (2005).

139 A. M. Bowen and R. G. Nuzzo, Adv. Funct. Mater. 19, 3243 (2009).

140 S. Lee, S. Kim, T. T. Kim, Y. Kim, M. Choi, S. H. Lee, J. Y. Kim and B. Min, Adv. Mater. 24, 3491 (2012).

141 H. Tao, A. Strikwerda, K. Fan, W. J. Padilla, X. Zhang and R. D. Averitt, in Lasers and Electro-Optics (CLEO) and Quantum Electronics and Laser Science Conference (QELS) (IEEE, 2010), pp. 1-2.

142 H. Tao, A. C. Strikwerda, K. Fan, W. J. Padilla, X. Zhang and R. D. Averitt, J. Infrared. Millim. Terahertz. Waves. 32, 580 (2011).

143 Y.-S. Lin, Y. Qian, F. Ma, Z. Liu, P. Kropelnicki and C. Lee, Appl. Phys. Lett. 102, 111908 (2013).

144 M. Lapine, D. Powell, M. Gorkunov, I. Shadrivov, R. Marques and Y. Kivshar, Appl. Phys. Lett. 95, 084105 (2009).

145 C. Kurter, P. Tassin, L. Zhang, T. Koschny, A. P. Zhuravel, A. V. Ustinov, S. M. Anlage and C. M. Soukoulis, Phys. Rev. Lett. 107, 043901 (2011).

146 H. Němec, P. Kužel, F. Kadlec, C. Kadlec, R. Yahiaoui and P. Mounaix, Phys. Rev. B. 79, 241108 (2009).

147 D. Nykypanchuk, M. M. Maye, D. van der Lelie and O. Gang, Nature 451, 549 (2008). 